\title{
Optical model for multilayer glazing systems: Experimental validation through the analytical prediction of encapsulation-induced variation of PV modules efficiency
}

\author{
Maider Machado $^{1}{ }^{*}$, Tomás Baenas², Naiara Yurrita ${ }^{1}$ \\ 1 TECNALIA. Energy and Environment Division. Paseo Mikeletegi, 2. 20009-San Sebastián \\ (Spain). \\ 2 University of Alicante. Applied Mathematics Department. Carretera de S. Vicente del Raspeig, \\ s/n. 03690, S. Vicente del Raspeig, Alicante (Spain). \\ *Corresponding author: maider.machado@tecnalia.com
}

A simple analytical calculation based on a transfer matrix method for incoherent optics, allowing the prediction of photovoltaic module efficiencies in different encapsulation conditions is presented. This approach is used for the experimental validation of the main features of the optical model for multilayer glazing systems considered, through the relation between the external quantum efficiency of the module and its optical modelling. The theoretical procedure avoids the need to manufacture and characterize by solar simulator or external quantum efficiency measurements all the variety of photovoltaic module configurations, which is of interest at research and manufacturing levels, especially for building-integrated photovoltaics.

The absorptivity of encapsulated solar cells is not directly accessible from direct airbare cell or air-encapsulated cell optical measurements, and therefore analytical or numerical methods are generally needed. The calculations presented in this work provide closed analytical expressions for the layer-by-layer absorption of the different components of a photovoltaic module. From a small set of experimental measurements of a particular encapsulation configuration, and the theoretical expressions for spectral absorptivities, the short-circuit current of a module can be predicted for any other encapsulation scheme. It will be proved that the method accurately matches shortcircuit current density of the modules as obtained from experimental measurements. Results will be presented for crystalline silicon and CIGS thin film cell technologies with several glass and encapsulation material combinations.

Keywords: Optical model; Encapsulation; Absorptivity; Short-circuit current.

\section{Introduction}

The theoretical problem of the distribution of the short-wave absorptivity inside an incoherent optical system with planoparallel optical interfaces, with application to glazing systems energy performance, has been recently revisited by Baenas and Machado (2016). This approach is based in a convenient use of the classical matrix transfer methods, limited to the usual modeling features of the standards (ISO 9050, 
ISO 15099, etc.) which allows obtaining closed analytical expressions for the shortwave $^{1}$ energy magnitudes (transmittance, reflectance, absorptance) of the optical system. From these magnitudes it is possible to achieve, by analytical inversion, the experimental characterization of the optical components and the calculation of the layer-by-layer absorption (through energy fluxes calculation), from the study of certain reference system configurations. Then, the obtained results can be extrapolated to more complex cases (glazing systems with additional components, for instance). The details of such a procedure can be found in Baenas and Machado $(2009,2016)$.

For the purpose of this work, the case study of opaque systems, i.e. those incorporating a component with null transmissivity, is of special interest. As will be discussed herein, the related transfer matrix is not well-defined, due to zero divisors. Then, the internal reflectivity in the interface of such type of optical component may be obtained by means of singular solutions (not included in the general solution of a multilayer system, but having full physical sense) of the algebraic equation system describing the short-wave energy magnitudes of the optical system. The absorptivity of the opaque component is then obtained as a function of this reflectivity. The corresponding equations were obtained in summary form by Baenas and Machado (2016), for a typical photovoltaic (PV) module (encapsulation materials - glass and polymer - and opaque PV cell).

It should be noted that this procedure of obtaining the absorptivity of an opaque component, in the particular case of an encapsulated PV cell, also allows an indirect experimental verification of the referenced optical model. In this regard, the model follows a physical description and approach for the iterative calculation of the incoming and outgoing fluxes which is equivalent to that of the classic literature (Edwards, 1977; van Dijk and Goulding, 1996; Siegel, 1973; Shurcliff, 1974; Wijeysundera, 1974; van Nijnatten, 1994; Wright, 1998; i.a.). The procedure is also equivalent to the one followed by all the international standards dealing with this issue (mainly ISO 15099, EN 13363). The main differences are the matrix formalism used (for a net radiation method) and the utilization of the mentioned singular solutions.

To our best knowledge, a direct measurement of the layer-by-layer absorption within a multilayer glazing system is not feasible. Related standards rely on numerical procedures that have been accepted with a basis on their accordance with derived effects like the thermal balances (Finlayson et al., 1993; van Dijk and Goulding, 1996; among others). In contrast, the experimental validation proposed herein is based in the relation between the internal absorption and the conversion efficiency of a photovoltaic module, through the short-circuit current density of the module. In this sense, this work involves firstly a verification of the main features of the referenced optical model, followed by the proposal of an effective procedure for the prediction of encapsulationinduced variation of PV modules efficiency, as described below.

${ }^{1}$ Within this framework, short-wave radiation commonly refers to near-ultraviolet (UV), visible (Vis) and near-infrared (NIR) spectra. 
The design phase of PV modules, especially in the case of building-integrated photovoltaic (BIPV) modules, implies the consideration of a wide range of possible configurations complying with PV and architectural requirements. Multiple laminated module configurations may be conceived in terms of cover material and polymeric interlayer type, thickness and even color. The manufacturing and experimental characterization of the optical and electrical properties of each configuration is therefore costly and time demanding. The use of a direct calculation method to predict the variation in modules efficiency for any change in the encapsulation conditions may represent significant savings for the manufacturing industry.

The short-circuit current density of a PV module, and therefore its efficiency for energy conversion, is directly related with the spectral absorptivity of the PV cell in encapsulated conditions. This magnitude differs from the absorptivity in air-bare cell configuration, due to the different refractive indexes of the surrounding media involved, and the corresponding change of reflectivity in the interface. While the reflectivity (and therefore the absorptivity) of a bare cell is directly accessible through spectrophotometric measurements, the absorptivity of the cell once it is encapsulated cannot be directly measured and must be predicted from simulation.

A simple analytical model is presented here as a tool to predict the variation in PV modules efficiency induced by modifications in the cells encapsulation and cover materials with planar structure, just from a reduced set of experimental measurements of a reference encapsulation scheme. The method is particularly valid, therefore, for PV modules optimization. The consideration of a planar structure is in line with, e.g., Krauter and Hanitsch (1996), or Lu and Yao (2007) (both based in ray-tracing method) in order to obtain closed expressions for cell absorptivity.

When other features are introduced in the modelling, such as optical confinement due to the presence of a back surface reflector (BSR) and light trapping effect (see e.g. Hylton (2006), for a comprehensive study of this issue), numerical techniques or computational methods are needed to obtain the energy magnitudes of the system. These comprise from the ideal Lambertian cases of light trapping schemes, including perfect diffusely scattering surface textures and analytical formulation (Goetzberger, 1981, Shimokawa et al., 1996, Green, 2002), to the use of a wide variety of simulation methods.

Khoo et al. (2012) used a simple experimental and analytical procedure to characterize the parasitic absorptances of the encapsulation material in a particular PV module, but no optical model is proposed to predict cell absorptivity and short-circuit current. PV Optics software by Sopori et al. (1999) makes use of a combination of wave optics and ray-tracing techniques to calculate the optical properties of thin-film and wafer-based cells and modules. Santbergen and van Zolingen (2008) proposed a multidirectional numerical net radiation method to calculate spectral and integrated absorptivities of 
bare and encapsulated silicon cells. The results of the model were then applied to estimate the fraction of solar radiation absorptance which is converted into heat and its effect on the temperature and energy yield of photovoltaic systems. A similar approach was followed by Santbergen et al. (2010) for several thin film photovoltaic technologies.

As an alternative to numerical approaches, the specific analytical expressions from the optical model of Baenas and Machado (2016) for the determination of the energy absorptance of encapsulated PV cells are applied in this paper to crystalline silicon and CIGS cells in combination with extraclear (low-iron), float and thin glass as covers, together with thermoplastic polyurethane (TPU) and ethyl vinyl acetate (EVA) as encapsulation materials. The results of the optical model are compared with the corresponding experimental short-circuit current values as obtained from External Quantum Efficiency (EQE) measurements.

\section{Theoretical modeling}

Under some modeling hypothesis related with the incoherent superposition of shortwave radiation and the aim to describe the energy performance, the analytical study of the propagation of solar radiation through planoparallel optical systems avoids the need to use complex numerical methods to solve the equations of Optics for the modeling of multiple glazing systems, laminated glass, PV modules or the like. Within this framework, closed analytical expressions for the short-wave energy magnitudes (transmittance, reflectance, absorptance) have been previously provided in Baenas and Machado $(2009,2016)$, with a basis on the classical transfer matrix methods (Harbecke, 1986; van Nijnatten, 1994; Pfrommer et al., 1995; Maestre, 2000; i.a.). These expressions do not include angle-dependent optical properties (Rubin, 1982; Rubin et al., 1998; Furler, 1991; van Nijnatten, 2001; Maestre et al., 2006; i.a.), light diffusing or shading elements (Finlayson et al., 1993).

The optical model applied herein is based on the separation of a real optical glazing system in optical components (interfaces, glass substrates, films and coatings, mainly), which are defined through a transfer matrix connecting short-wave energy fluxes (irradiance or incoming energy flux, and radiosity or outcoming one) on each side of the component.

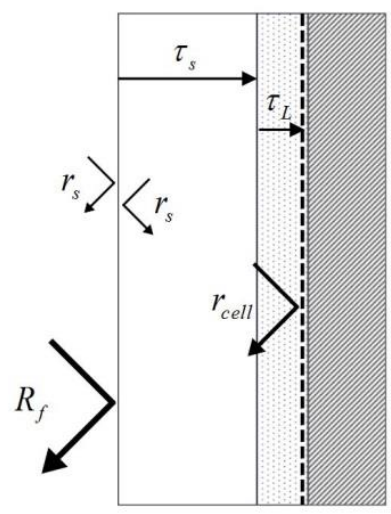


Fig. 1: Schematics of the optical system

Fig. 1 shows a diagram of the optical system structure and the spectral magnitudes involved in the calculation. The equations provided in this work are valid for a laminated PV module consisting in a superstrate, typically uncoated glass, a polymeric encapsulation material with refractive index very similar to that of the superstrate, and an opaque PV cell. Systems involving coated superstrates or different refractive indexes for superstrate and encapsulation material (which would imply additional internal reflectivities) can be treated within the general formalism used, but the corresponding mathematical expressions should be specifically derived.

Following Baenas and Machado (2016), the opaque cell may be described within this formalism by means of a virtual internal coating component, which allows the introduction of (front) reflectivity inside the system $\left(r_{\text {cell }}\right)$, shown in Fig. 1. This magnitude is intrinsically associated to the cell, so that once it has been determined for a specific encapsulation scheme, it can be used for the calculation of any other configuration of the same type of cell and similar refractive index of superstrate and polymer film. It can be shown (Rubin, 1982; Baenas and Machado, 2016) that typical polymeric encapsulation materials have close refractive indexes, also similar to those of, e.g., soda-lime glass substrates.

The reflectivity of the encapsulated cell is obtained from a singular solution, related to null transmissivity, of a general equation system for the energy coefficients of the optical system: transmittance $(T)$, front $\left(R_{f}\right)$ and back $\left(R_{b}\right)$ reflectances. In the case of an uncoated superstrate and an opaque substrate, $T=0$ and $R_{b}$ do not participate in the optical problem formulation to obtain $r_{\text {cell }}$. The quoted algorithm leads to a transfer matrix for the PV module given by the product of the transfer matrix of each component,

$$
M_{P V}\left(T, R_{f}, R_{b}\right)=M_{[}\left(r_{s}\right) M_{S}\left(\tau_{s}\right) M_{]+[}\left(\tau_{L}\right) M_{(}\left(r_{c e l l}\right) M_{P}\left(\tau_{2}\right) M_{]}\left(R_{b}\right),
$$

where the symbolic subscripts and transfer matrices are those defined in Table 1 of Baenas and Machado (2016). Here, $M_{P}\left(\tau_{2}\right)$ describes the opaque component and $M_{(}\left(r_{\text {cell }}\right)$ transfer matrix stands for the virtual internal coating, which can be considered, without loss of generality, as a simple interface, with transmissivity $1-r_{\text {cell }}$

$M_{(}\left(r_{\text {cell }}\right)=\frac{1}{1-r_{\text {cell }}}\left(\begin{array}{cc}1 & -r_{\text {cell }} r_{(, b} \\ r_{\text {cell }} & \left(1-r_{\text {cell }}\right)^{2}-r_{\text {cell }} r_{(, b}\end{array}\right), \quad M_{P}\left(\tau_{2}\right)=\frac{1}{\tau_{2}}\left(\begin{array}{cc}0 & 1 \\ \tau_{2}^{2} & 0\end{array}\right)$.

Back reflectivity of the virtual coating, $r_{(, b}$, is not defined in the opaque system, and consistently, does not have any influence on it. A singular solution of the equation 
system for the energy transmittance and reflectances, obtained from $M_{P V}$ matrix elements,

$$
T=\left(M_{P V}\right)_{12}^{-1}, \quad R_{f}=\left(M_{P V}\right)_{22}\left(M_{P V}\right)_{12}^{-1}, \quad R_{b}=-\left(M_{P V}\right)_{11}\left(M_{P V}\right)_{12}^{-1},
$$

is needed since transfer matrix $M_{P}$ is not mathematically defined for $\tau_{2}=0$. This problem is avoided by substituting $M_{P V}$ by its limit when $\tau_{2}$ approaches 0 , $\lim _{\tau_{2} \rightarrow 0^{+}} M_{P V}$, which is justified by the physical hypothesis of continuity of the energy coefficients. Then, the equation for the front reflectance of the system is

$$
R_{f}=\lim _{\tau_{2} \rightarrow 0^{+}} \frac{\left(M_{P V}\right)_{22}}{\left(M_{P V}\right)_{12}}=r_{S}+\frac{\tau_{S}^{2} \tau_{L}^{2} r_{c e l l}\left(1-r_{S}\right)^{2}}{1-r_{S} r_{c e l l} \tau_{S}^{2} \tau_{L}^{2}}
$$

So that the spectral reflectivity of the encapsulated PV cell, $r_{c e l l}$, is simply given by

$$
r_{c e l l}=\frac{R_{f}-r_{S}}{\tau_{S}^{2} \tau_{L}^{2}\left(1-2 r_{S}+R_{f} r_{S}\right)}
$$

which allows its experimental characterization from a set of usual reference system configurations. Summarizing, $R_{f}$ is the front reflectance of the system, $r_{s}$ is the airsuperstrate reflectivity, $\tau_{S}$ is the transmissivity of the superstrate and $\tau_{L}$ is the transmissivity of the polymer film. The procedure to obtain each of these magnitudes from experimental characterization is described in detail in Baenas and Machado (2009) and references therein, and involves the experimental (spectrophotometric) measurement of $R_{f}$, the measurement of transmittance and reflectance of the superstrate (and subsequent calculation of $\tau_{s}$ and $r_{s}$ ), and the measurement of transmittance of a laminated glass with the involved superstrates and polymeric film (and subsequent calculation of $\tau_{L}$ ).

As stated above, the used theoretical model does not include angle-dependent optical properties. Coatings used in glazing systems are multilayered thin films with coherent optical behavior. Therefore, Fresnel equations can be formulated by means of the characteristic matrices theory (e.g. Epstein, 1952; Heavens, 1960; Thelen, 1989 among others), provided that the whole multilayered structure is known (at least through its complex refractive indexes and layer thicknesses), in order to obtain the angular properties of the coating. Given that this information is not always available (e.g., protected by property rights or considering the current regulatory framework scope), several semi empirical approaches have been developed aiming to obtain a reasonable prediction of the directional optical properties from the near normal incidence case, for instance, Rubin et al. (1998) or van Nijnatten $(1999,2001)$.

However, when coated components are not considered within the modelling, as is the case for the systems exemplified herein, the optical performance at non-normal 
incidence is directly obtained from Fresnel equations for the incoherent case, and Snell law, see e.g. Furler (1991) for glazing systems or Krauter and Hanitsch (1996) or Lu and Yao (2007) for PV modules, among others. In this situation, the substrate-type transmissivities $\tau$ ( $\tau_{s}$ and $\tau_{L}$ ) from the previous expressions, must be substituted by $\tau^{1 / \cos \theta}, \theta$ being the irradiation incidence angle $(\theta=0$ for normal incidence), which take into account the different light pathlengths within the layered media. Note that in a standard PV module with antireflective coatings in the upper cell surface, this substitution in the transmissivity can also be assumed as a good approximation for the usual values of the refractive indices of the components (Yamada et al. 2001, SjerpsKoomen et al. 1996).

Any light trapping mechanism present within the system is quantified by $r_{\text {cell }}$ reflectivity, when this magnitude is obtained from the experimental reflectance of the module. However, its influence cannot be isolated in the total value of the cell reflectivity because this type of mechanism is not considered in the theoretical modeling.

The determination of closed analytical expressions for the layer-by-layer absorption in a PV module follows the iterative procedure described in Baenas and Machado (2016), which is a specific adaptation to matrix formalism of the classical method in Siegel (1973) and Wright (1998) among others (see introduction), and standardized in ISO 15099. The corresponding analytical expressions for the absorptance of the whole system (photovoltaic module, $\left.A_{\text {mod }}\right)$, opaque encapsulated PV cell $\left(A_{c e l l}\right)$ and the glassencapsulation film $\left(A_{\text {enc }}\right)$, are given by

$$
\begin{gathered}
A_{\text {mod }}=\left(1-r_{s}\right) \frac{1-r_{\text {cell }} \tau_{s}^{2} \tau_{L}^{2}}{1-r_{s} r_{\text {cell }} \tau_{s}^{2} \tau_{L}^{2}}, \\
A_{\text {cell }}=\left(1-r_{s}\right)\left(1-r_{\text {cell }}\right) \frac{\tau_{s} \tau_{L}}{1-r_{s} r_{\text {cell }} \tau_{s}^{2} \tau_{L}^{2}}, \\
A_{\text {enc }}=\left(1-r_{s}\right)\left(1-\tau_{s} \tau_{L}\right) \frac{1+\tau_{s} \tau_{L} r_{\text {cell }}}{1-r_{s} r_{\text {cell }} \tau_{s}^{2} \tau_{L}^{2}},
\end{gathered}
$$

consistently verifying that $A_{\text {mod }}=1-R_{f}=A_{\text {cell }}+A_{\text {enc }}$.

The absorptivity of the PV cell can be directly related with the short-circuit current of the module. The general expression for the short-circuit current density of a bare PV cell is (ASTM E1021-15, Hartman et al., 1982),

$$
J_{\text {sc,bare }}=q \int_{\lambda_{1}}^{\lambda_{2}} \phi(\lambda) E Q E_{\text {bare }}(\lambda) d \lambda,
$$


where $q$ is the elementary charge, $\phi(\lambda)$ is the incoming spectrum photon flux (ASTM G173-03 discretized global spectral distribution has been used in this work) and $\lambda_{1}, \lambda_{2}$ define the relevant wavelength range for the determination of the short-circuit current. $E Q E(\lambda)$ is the External Quantum Efficiency function of the cell, defined as the ratio of charge carriers collected by the solar cell to the number of photons of a given wavelength incident on the cell. Once the cell is encapsulated, the incoming photon flux is modified by the additional reflectivities and absorptivities introduced by the encapsulation materials. Experimentally, the short-circuit density of the module can be obtained, analogously to (7), from the EQE measured for the encapsulated cell, denoted as $E Q E_{\text {mod }}(\lambda)$,

$$
J_{s c, \text { mod }}=q \int_{\lambda_{1}}^{\lambda_{2}} \phi(\lambda) E Q E_{\text {mod }}(\lambda) d \lambda
$$

For the theoretical description, it is more convenient in this case to express the external quantum efficiency in terms of the Internal Quantum Efficiency (IQE), which is the ratio of charge carriers collected by the solar cell to the number of photons absorbed by the cell,

$$
I Q E_{\text {mod }}(\lambda)=\frac{E Q E_{\text {mod }}(\lambda)}{A_{\text {cell }}(\lambda)}
$$

considering that $A_{\text {cell }}$ stands for the absorptivity of the PV cell in encapsulation conditions. The IQE function of a bare cell, $I Q E_{\text {bare }}(\lambda)$, is easily determined from aircell external quantum efficiency and reflectance measurements, given the fact that $A_{\text {bare }}=1-R_{f, \text { bare }}$. It will be assumed here that this magnitude is intrinsic to the cell and does not vary for different encapsulation conditions (Khoo et al., 2012), so that $I Q E_{\text {bare }}(\lambda)=I Q E_{\text {mod }}(\lambda)$. In this way, expressing Eq. (8) in terms of Eq. (9) leads to,

$$
J_{s c, \bmod }=q \int_{\lambda_{1}}^{\lambda_{2}} \phi(\lambda) I Q E_{\text {bare }}(\lambda) A_{\text {cell }}(\lambda) d \lambda \text {. }
$$

Here the short-circuit current density of the module is now given by the original incoming photon flux, the internal quantum efficiency of the cell measured in air, and the absorptivity of the cell in the particular encapsulation conditions, as obtained from Eq. (6), i.e, the optical model. Therefore, Eq. (10) allows theoretical predictions of $J_{s c, \text { mod }}$ for different module configurations. The accuracy of this model will be proved in this work, by comparing $J_{s c, \text { mod }}$ values from Eq. (10) with those obtained experimentally from measurements of the EQE of the whole module and Eq. (8). Additionally, the spectral matching between the integrands in Eq. (8) and Eq. (10) will be shown. 


\section{Materials and experimental methods}

Commercial 6" monocrystalline silicon (c-Si) cells from Sunways and $210 \times 100 \times 0.5 \mathrm{~mm}$ thin film CIGS cells from Global Solar have been used for this study. C-Si and CIGS cells have been combined with soda-lime float glass (Planilux TM $4 \mathrm{~mm}$ from SaintGobain Glass), soda-lime extraclear glass (Diamant TM $4 \mathrm{~mm}$ from Saint Gobain Glass) and borosilicate thin glass (D 263 ${ }^{\mathrm{TM}} \mathrm{T} 30 \mu \mathrm{m}$ from Schott) as transparent covers. All the combinations of these covers with TPU (EncapsolarTM PV 251 Stevens Urethane, 0.49 $\mathrm{mm}$ ) and EVA (PV135A TM Stevens Urethane, $0.46 \mathrm{~mm}$ ) as encapsulation materials have been manufactured and experimentally measured.

The samples needed for this study are either glass-glass or glass-cell laminates. A vacuum laminator was used for the encapsulation process, using standard lamination cycles.

Transmittance and reflectance spectra were measured using a UV/vis/NIR spectrophotometer (JASCO V-670) equipped with a $150 \mathrm{~mm}$ integrating sphere, calibrated in the wavelength range $300-2200 \mathrm{~nm}$. The bare and laminated cells were characterized by external quantum efficiency measurements obtained with a Bentham PVE300 spectral response system under unbiased light. In all cases three measurements were taken for each sample, and the resulting spectra were averaged, within the repeatability limit (ISO 5725-6).

\section{Results and discussion}

Figs. 2 and 3 show the experimental absorptivity (obtained from total reflectance measurements as $A(\lambda)=1-R(\lambda)$, given that $T(\lambda)=0)$ for bare crystalline silicon and CIGS cell respectively, together with the calculated spectral absorptivity for the same cells in several encapsulation conditions: extraclear, float and thin glass covers with EVA and TPU encapsulation materials. A single $r_{\text {cell }}$ reflectivity spectrum has been used for all the encapsulation configurations in order to exemplify the predictive capability of the method. For the calculations presented herein, cell reflectivity has been obtained from Eq. (5) for the case of extraclear glass and EVA.

In the case of the silicon cell (Fig. 2), encapsulation leads to an overall decrease of the absorptivity values, the best performance provided by encapsulation with thin glass. In the case of the CIGS cells and encapsulation materials used for this study (Fig. 3), there is a better optical coupling due to encapsulation in a wavelength range approximately between 400 and $860 \mathrm{~nm}$ for extraclear glass and between 440 and $600 \mathrm{~nm}$ for float glass and EVA. These ranges where optical coupling is enhanced are however reduced to $400-600 \mathrm{~nm}$ for extraclear glass and TPU encapsulation material. Thin glass provides the best performance, with enhanced absorptivity with respect to bare cell in a wavelength range from 400 to $1000 \mathrm{~nm}$, covering most of the active range for PV production. 
Between 300 and $380 \mathrm{~nm}$ (ultraviolet wavelength range), the optical procedure to characterize the encapsulation material includes several error sources (Baenas and Machado, 2009) due to the low transmissivity of the polymers and the use of an integrating sphere for reflectance measurements, which generally provide lower accuracy in this spectral range. Therefore, this spectral range has not been considered in the theoretical prediction of the absorptivity of the encapsulated cell. For the purpose of computing the short-circuit current density, this contribution is only around $0.1 \%$.

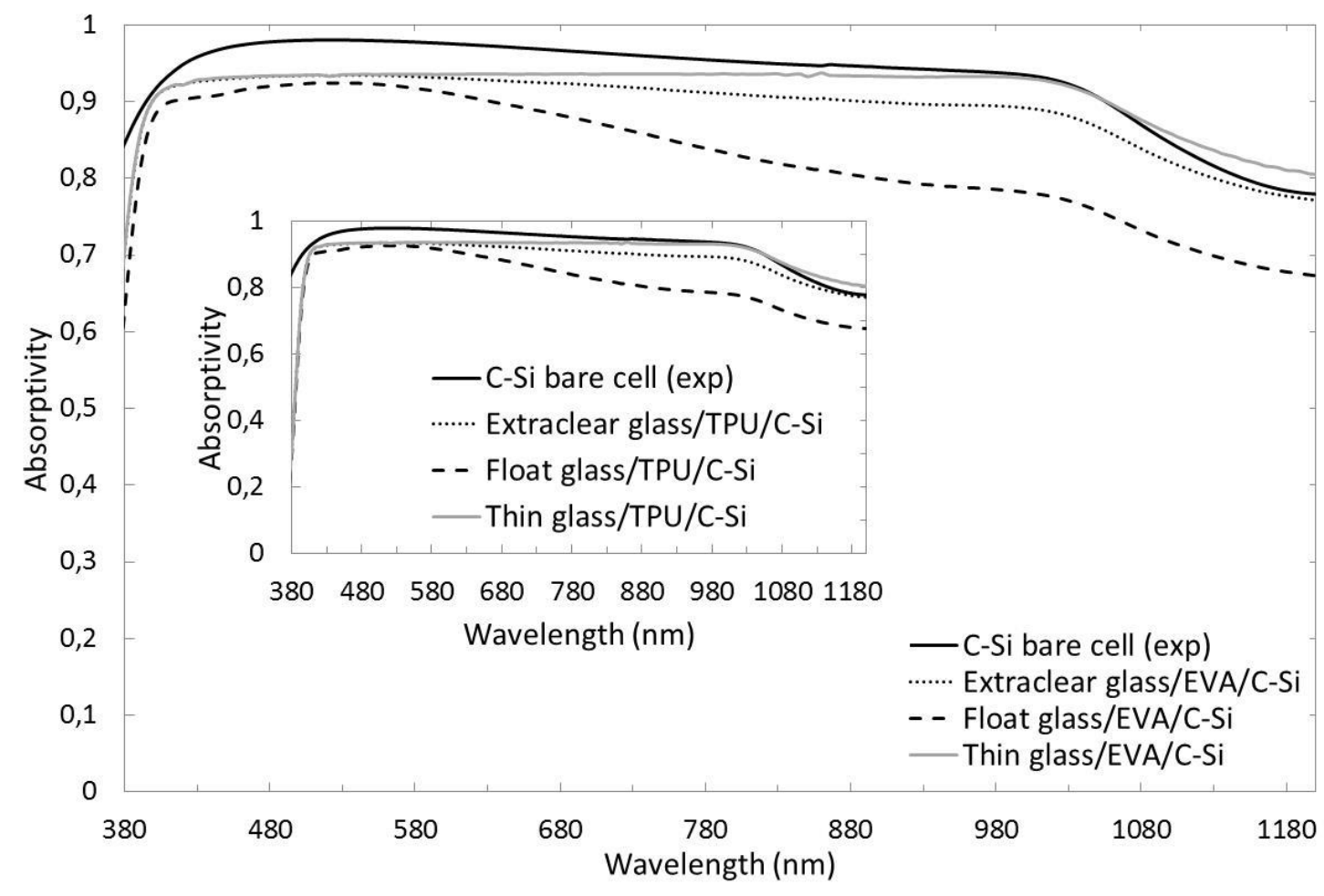

Fig. 2: Experimental absorptivity of a bare crystalline silicon cell and calculated absorptivities of encapsulated silicon cells (EVA in main graph and TPU in insert). 


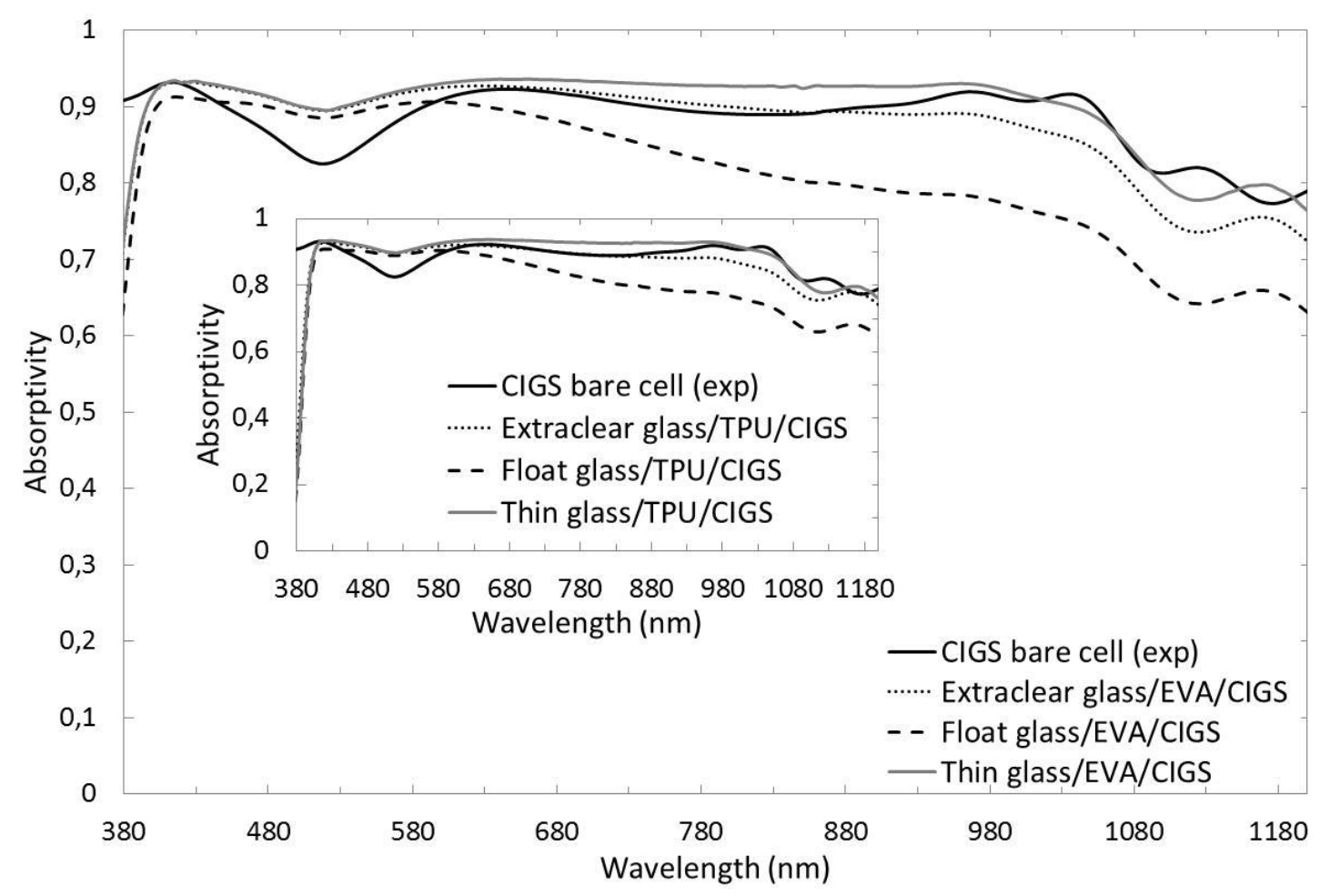

Fig. 3: Experimental absorptivity of a bare CIGS cell and calculated absorptivities of encapsulated CIGS cells (EVA in main graph and TPU in insert).

The measured EQE of all the encapsulated modules considered for this study, used for the determination of experimental short-circuit density values, is shown in Figs. 4 and 5. These curves show the same trends as the calculated absorptivity curves. The overall decrease in absorptivity for encapsulated silicon cells (Fig. 2) corresponds with a lower EQE in the same spectral ranges. Analogously, the improved optical coupling induced by encapsulation in several wavelength ranges observed in Fig. 3 for CIGS leads to enhanced EQE in the same wavelength ranges. 


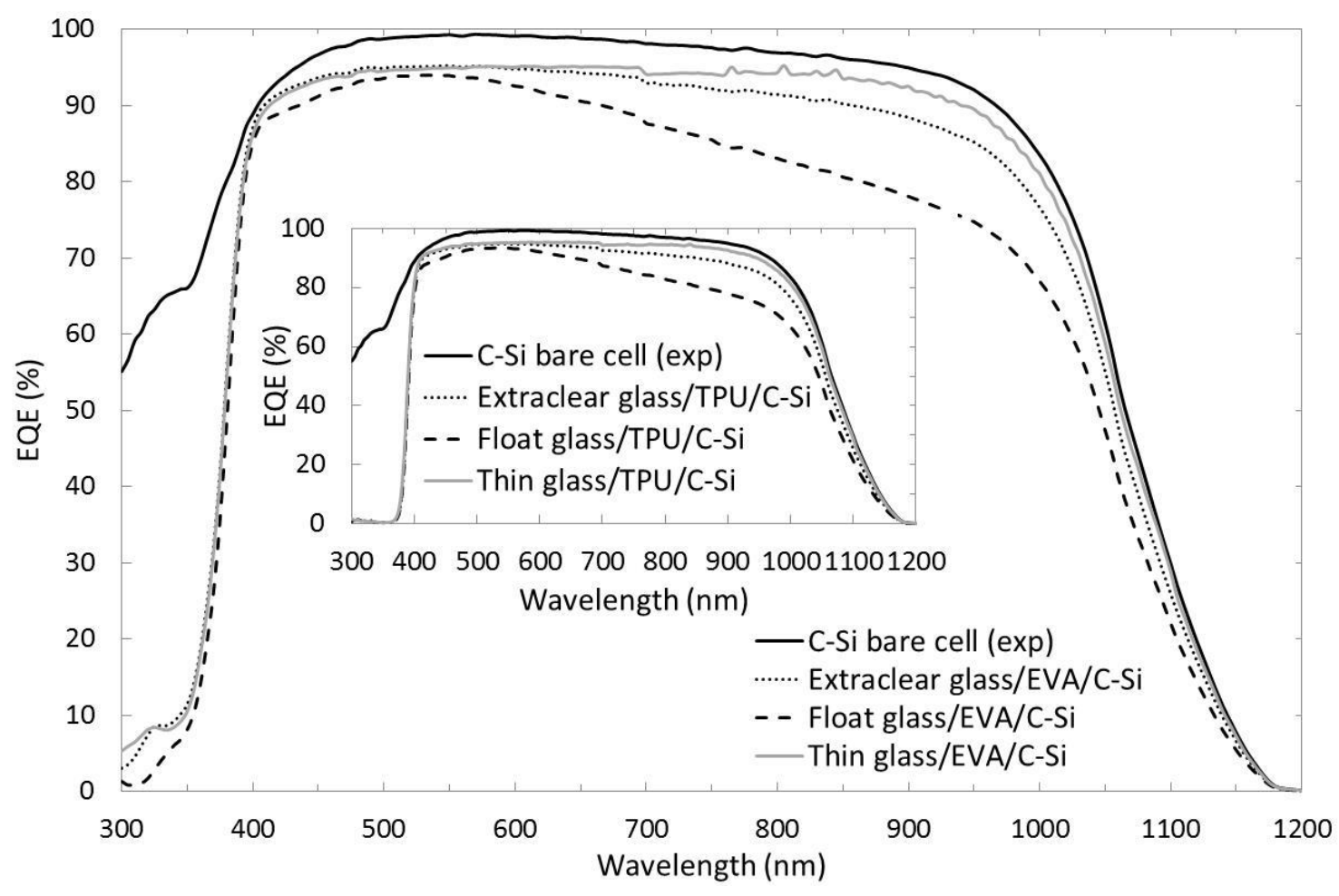

Fig. 4: Experimental EQE curves for bare and encapsulated silicon cells (EVA in main graph and TPU in insert).

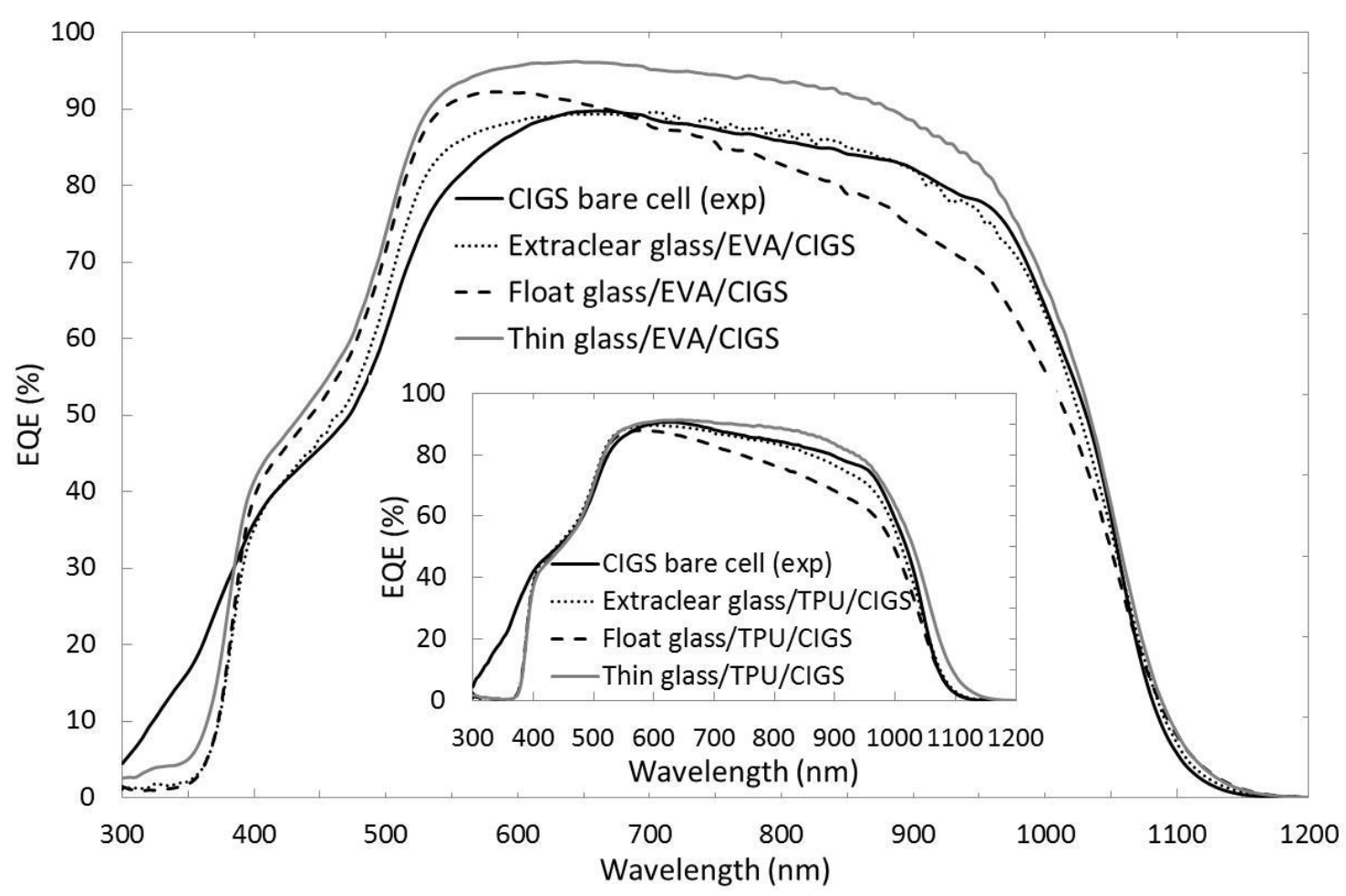

Fig. 5: Experimental EQE curves for bare and encapsulated CIGS cells (EVA in main graph and TPU in insert). 
Tables 1 and 2 compare the values of the short-circuit current density calculated using the optical model - Eqs. (5), (6) for $A_{\text {cell }}$ and (10) - with the experimental values from EQE measurements - Eq. (8) - for c-Si and CIGS cells. The results show that the method provides short-circuit current density values in very good agreement with the experimental data.

For EVA encapsulation material and Si cells the method gives excellent agreement with experiment with all the superstrates used. Slightly larger deviations are found for encapsulation with TPU, in the case of extraclear and float glass. It has been verified that the calculation of $r_{\text {cell }}$ parameter with a TPU encapsulation leads to negligible differences in the obtained $J_{s c}$ values, as expected. The higher error is suspected to be related to a difference in the flowing of TPU over the samples edge during direct lamination on the cells, in comparison with the overflowing in glass-glass configurations used for the initial experimental characterization. This would lead to different TPU layer thickness in the modules than in the glass-glass samples used to determine the polymer transmissivity $\left(\tau_{L}\right)$, which is later used for the calculations. These differences are therefore due to experimental issues, and they are not related with the calculation procedure.

In the case of CIGS cells, EVA combinations with extraclear and float glass show an excellent agreement. Thin glass and EVA, together with all TPU combinations, show higher errors than those found for Si cells. It should be noted that a lower homogeneity has been experimentally evidenced in the commercial CIGS cells used, which necessarily leads to small deviations.

However, it can be said that for the purpose of research or manufacturing activities, the method is highly useful to predict the variation in cell efficiency (which in general correlates with the variation in short-circuit current density). In the most unfavorable case, the relative difference between theory and experiment is $1.5 \%$, which is related to a difference of $0.5 \mathrm{~mA} / \mathrm{cm}^{2}$ in a short-circuit current density of $34.4 \mathrm{~m} / \mathrm{cm}^{2}$. Note that in several cases (mainly for EVA), the relative difference is less than $1 \%$ and even null $(0.0 \%)$. In addition to this, $\Delta J_{s c}$ theory-experiment differences - which include modeling features and experimental errors and limitations - are an order of magnitude lower than differences between different encapsulation conditions, justifying the use of the method for the prediction of short-circuit current density values.

Fig. 6 depicts the integrands of Eqs. (8), experimental, and (10), calculated, for the case of silicon cells encapsulated with extraclear glass and EVA, showing that a very good agreement is also found spectrally. As an insert, calculated and experimental EQE curves for the same system are shown.

Note that Fig. 6 shows a modification in the behavior of calculated and experimental curves around $900 \mathrm{~nm}$, changing from a lower value of the calculated integrand with respect to the experimental one, to the inverse situation. This is probably due to the transition from the opaque regime of the cell (incoming light on the cell is completely 
absorbed before reaching the BSR) to the transparent one (there exists absorption in the BSR). See, e.g., Santberger and van Zolingen (2008), where the same theoretical vs experimental behavior is shown. This effect introduces a small error in $A_{\text {cell }}$ magnitude, since this parameter includes the whole absorptivity of the cell, while only the part due to the active semiconductor material of the cell should be considered in order to compute the IQE (Khoo et al. 2012). 
Table 1: Calculated and experimental values of short-circuit current density of several encapsulation schemes for silicon cells.

\begin{tabular}{|c|c|c|c|c|}
\hline Encapsulation configuration & $\begin{array}{r}J_{s c} \text { calc. } \\
\left(\mathrm{mA} / \mathrm{cm}^{2}\right)\end{array}$ & $\begin{array}{r}J_{s c} \exp . \\
\left(\mathrm{mA} / \mathrm{cm}^{2}\right)\end{array}$ & $\begin{array}{l}\Delta J_{s c} \text { total } \\
\left(\mathrm{mA} / \mathrm{cm}^{2}\right)\end{array}$ & $\begin{array}{r}\text { Error } \\
(\%)\end{array}$ \\
\hline Extraclear glass/EVA/Si cell & 37.3 & 37.2 & 0.1 & 0.2 \\
\hline Float glass/EVA/Si cell & 35.0 & 34.8 & 0.2 & 0.6 \\
\hline Thin glass/EVA/Si cell & 38.0 & 38.0 & 0.0 & 0.0 \\
\hline Extraclear glass/TPU/Si cell & 37.1 & 36.8 & 0.3 & 0.8 \\
\hline Float glass/TPU/Si cell & 34.9 & 34.4 & 0.5 & 1.5 \\
\hline Thin glass/TPU/Si cell & 37.9 & 37.9 & 0.0 & 0.0 \\
\hline
\end{tabular}

Table 2: Calculated and experimental values of short-circuit current density of several encapsulation schemes for CIGS cells.

\begin{tabular}{lrrrr} 
Encapsulation configuration & $\begin{array}{r}\boldsymbol{J}_{\boldsymbol{s c}} \text { calc. } \\
\left(\mathbf{m A} / \mathbf{c m}^{\mathbf{2}}\right)\end{array}$ & $\begin{array}{r}\boldsymbol{J}_{\boldsymbol{s c}} \mathbf{e x p} \\
\left(\mathbf{m A} / \mathbf{c m}^{2}\right)\end{array}$ & $\begin{array}{r}\boldsymbol{\Delta}_{\boldsymbol{s c}} \text { total } \\
\left(\mathbf{m A} / \mathbf{c m}^{2}\right)\end{array}$ & $\begin{array}{r}\text { Error } \\
\mathbf{( \% )}\end{array}$ \\
\hline Extraclear glass/EVA/CIGS cell & 31.3 & 31.3 & 0.0 & 0.0 \\
Float glass/EVA/CIGS cell & 29.4 & 29.4 & 0.0 & 0.0 \\
Thin glass/EVA/CIGS cell & 31.8 & 32.2 & -0.4 & -1.3 \\
Extraclear glass/TPU/CIGS cell & 30.9 & 30.5 & 0.4 & 1.4 \\
Float glass/TPU/CIGS cell & 29.1 & 28.7 & 0.4 & 1.2 \\
Thin glass/TPU/CIGS cell & 31.8 & 32.2 & -0.4 & -1.1
\end{tabular}




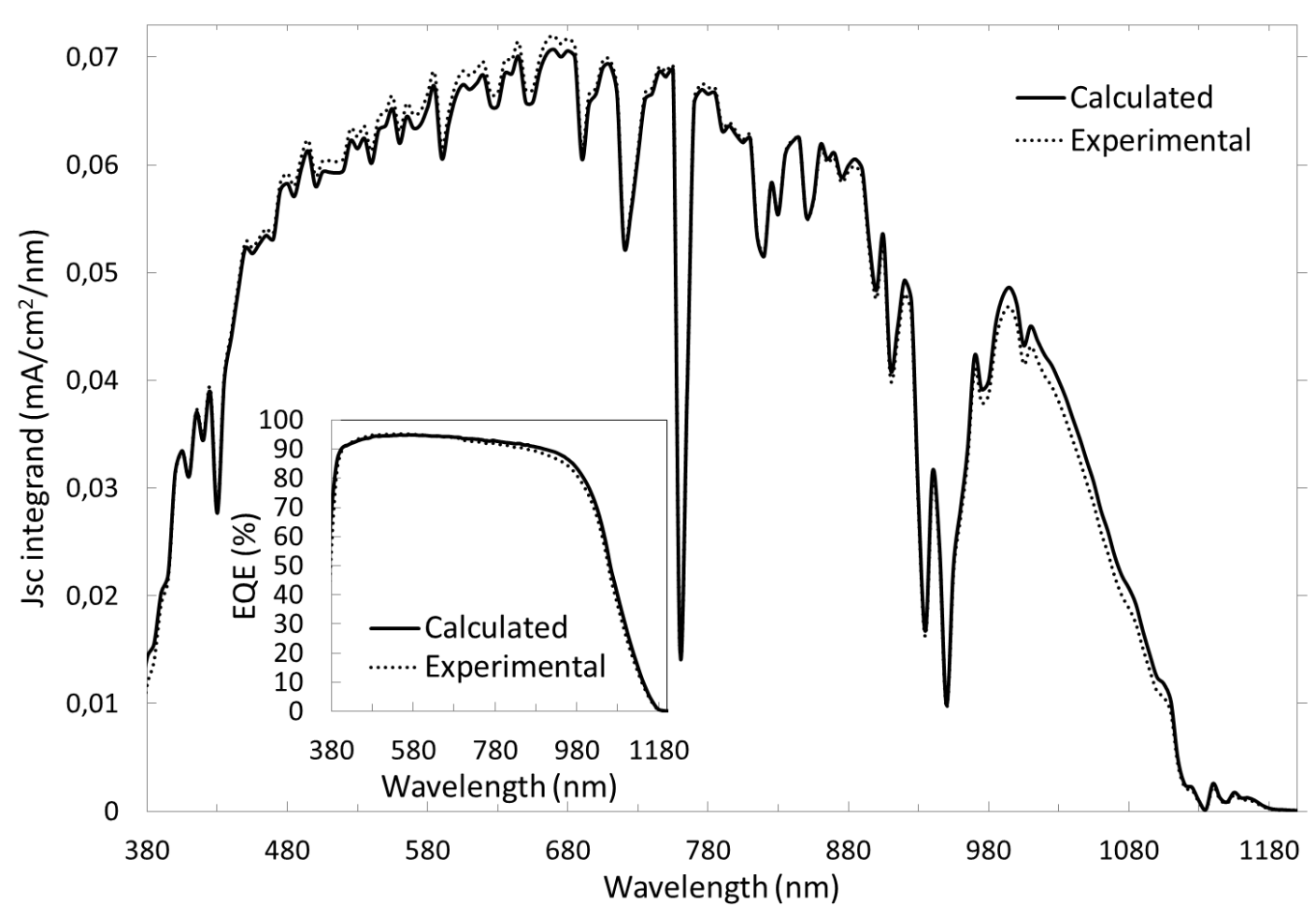

Fig. 6: Integrands of Eqs. (8) and (10), obtained from experimental and calculation procedures. Comparison between simulated and experimental EQE curves is shown as an insert.

\section{Conclusions}

An optical model based on transfer matrix method, providing closed analytical expressions for the short-wave absorptivity of an encapsulated solar cell, has been used to predict short-circuit current density values for different encapsulation conditions. The results of the optical model have been compared with those obtained from direct experimental determination of the external quantum efficiency of the modules. In addition to the predictive character of the method, an experimental validation of the main features of the optical model has been performed with a basis on the relationship between the photovoltaic module efficiency and the optical modeling of its cover materials. Such an experimental validation is a straightforward alternative to the use of thermal measurements (e.g. center-glass surface temperatures), which requires a complete thermal modelling and accurately controlled environmental conditions.

All the encapsulation combinations involving extraclear (low-iron), float and thin glass as superstrates, EVA and TPU as encapsulation materials with crystalline silicon and thin film CIGS solar cells have been calculated and measured. A very good agreement has been shown for both spectral and integrated magnitudes. The optical method can thus be reliably considered for the accurate determination of the influence of the encapsulation conditions on the electrical output of a photovoltaic module. 


\section{Acknowledgements}

This work has received funding from the European Union's Seventh Programme for research, technological development and demonstration under Grant Agreement No 609788.

\section{References}

ASTM E1021-15:2015 (International Standard). Standard Test Method for Spectral Responsivity Measurements of Photovoltaic Devices.

ASTM G173-03:2012 (International Standard). Standard Tables for Reference Solar Spectral Irradiances: Direct Normal and Hemispherical on $37^{\circ}$ Tilted Surface.

Baenas, T., Machado, M., 2009. Optical simulation of laminated glass. Proc. Glass Performance Days 2009, Ed. GPD-Glaston Finland Oy, pp. 742-745.

Baenas, T., Machado, M., 2016. Optical model for multilayer glazing systems: application to laminated glass and photovoltaic modules, Solar Energy 125, pp. 256266; doi:10.1016/j.solener.2015.12.027.

Edwards, D.K., 1977. Solar absorption by each element in an absorber-coverglass array. Solar Energy, 19, pp. 401-402

EN 13363-1:2003 (European Standard). Solar protection devices combined with glazing. Calculation of solar and light transmittance. Simplified Method.

Epstein, L.I., 1952. The Design of Optical Filters. J. Opt. Soc. Am., 42 (11), pp 806-810.

Finlayson, E.U., Arasteh D.K., Huizenga, C., Rubin M.D., and Reilly M.S., 1993. Window 4.0: Documentation of calculation procedures. Lawrence Berkeley Laboratory, University of California.

Furler, R.A., 1991. Angular Dependence of Optical Properties of Homogeneous Glasses. ASHRAE Transactions 97 (2).

Goetzberger, A., 1981. Optical confinement in thin Si solar cells by diffuse back reflectors. Proc. 15 $5^{\text {th }}$ IEEE Photovoltaic Specialists Conference, Orlando, pp. 867-870.

Green, M.A., 2002. Lambertian light trapping in textured solar cells and light-emitting diodes: analytical solutions. Prog. Photovolt: Res. Appl. 10, pp. 235-241; doi: $\underline{0.1002 / \text { pip.404. }}$.

Harbecke, B., 1986. Coherent and incoherent reflection and transmission of multilayer structures. Appl. Phys. B 39, pp. 165-170; doi: 10.1007/BF00697414. 
Hartman, J.S., Lind, M.A., Chaudiere, D.A., 1982. The sensitivity of calculated shortcircuit currents to selected irradiance distributions and solar cell spectral responses. Solar Cells, 6, pp. 133-148; doi: 10.1016/0379-6787(82)90062-X.

Heavens, O.S., 1960. Optical properties of thin films. Rep. Prog. Phys, 23, pp. 66-69; doi: $\underline{10.1088 / 0034-4885 / 23 / 1 / 301 .}$.

Hylton, J.D., 2006. Light coupling and light trapping in alkaline etched crystalline silicon wafers of solar cells. Ph.D. Thesis, University Utrecht, The Netherlands.

ISO 5725-6:1994 (International Standard). Accuracy (trueness and precision) of measurement methods and results - Part 6: Use in practice of accuracy values.

ISO 9050:2003 (International Standard). Determination of light transmittance, solar direct transmittance, total Solar Energy transmittance, ultraviolet transmittance and related glazing factors.

ISO 15099:2003 (International Standard). Thermal performance of windows, doors and shading devices - Detailed calculations.

Khoo, Y.S., Walsh, T.M., Lu, F., Aberle, A.G., 2012. Method for quantifying optical parasitic absorptance loss of glass and encapsulant materials of silicon wafer based photovoltaic modules, Solar Energy Materials and Solar Cells 102, pp. 153-158; doi:10.1016/j.solmat.2012.03.008.

Krauter, S., Hanitsch, R., 1996. Actual solar and thermal performance of PV-modules. Solar Energy Materials and Solar Cells 41-42, pp. 557-574.

Lu, Z.H., Yao, Q., 2007. Energy analysis of silicon solar cell modules based on an optical model for arbitrary layers. Solar Energy 81, pp. 637-647; doi:10.1016/j.solener.2006.08.014.

Maestre, I.R., 2000. Modelo óptico y térmico de acristalamientos complejos, Ph.D. Thesis (in spanish), University of Sevilla, Spain.

Maestre, I.R., Molina, J. L., Ross, A., Coronel, J.F., 2006. A single-thin-film model for the angle dependent optical properties of coated glazings. Solar Energy, 81, pp. 969-976; doi: 10.1016/j.solener.2006.12.002.

Pfrommer, P., Lomas, K. J., Seale, C., Kupke, Chr., 1995. The radiation transfer through coated and tinted glazing. Solar Energy, 54, pp. 287-299; doi:10.1016/0038$\underline{092 X(94) 00132-W .}$

Rubin, M., 1982. Solar optical properties of windows. Energy Research, 6, pp. 123-133; doi: $10.1002 /$ er.4440060204.

Rubin, M., Rottkay, K.V., Powles, R., 1998. Window Optics. Solar Energy, 62, pp. 149161; doi:10.1016/S0038-092X(98)00010-3. 
Santbergen, R., van Zolingen, R.J.C., 2008. The absorption factor of crystalline silicon PV cells: A numerical and experimental study. Solar Energy Materials and Solar Cells 92, pp. 434-444; doi:10.1016/j.solmat.2007.10.005.

Santbergen, R., Goud, J. M., Zeman, M., van Roosmalen, J.A.M., van Zolingen, R.J.C., 2010. The AM1.5 absorption factor of thin-film solar cells, Solar Energy Materials and Solar Cells 94, pp. 715-723; doi:10.1016/j.solmat.2009.12.010.

Shimokawa, R., Ishii, K., Takahashi, T., 1996. Optical confinement in thin films Si solar cells by diffuse reflective substrate. Jpn. J. Appl. Phys. 35, 1, 6A, pp. 3445-3456.

Siegel, R., 1973. Net radiation method for transmission through partially transparent plates. Solar Energy, 15, pp. 273-276; doi:10.1016/0038-092X(73)90090-X.

Sjerps-Koomen, E. A. , Alsema, E. A. , Turkenburg, W. C., 1996. A simple model for PV module reflection losses under field conditions. Solar Energy Vol. 57, No. 6, pp. 421432. doi:10.1016/S0038-092X(96)00137-5

Sopori, B., Madjdpour J., Zhang Y., Chen, W., 1999. PV Optics: an optical modeling tool for solar cell and module design, Proceedings of the International Symposium on Photovoltaics for the $21^{\text {st }}$ century 1999, vol. 99, p. 138.

Shurcliff, W.A, 1974. Transmittance and reflection loss of multi-plate window of a solar radiation collector: Formulas and tabulations of results for the case of $n=1.5$. Solar Energy, 16, pp. 149-154.

Thelen, A., 1989. Design of optical interference coatings. McGraw-Hill.

van Dijk, H.A.L., Goulding, J. (eds.), 1996. WIS reference manual, TNO Building and Construction Research.

van Nijnatten, P.A., 1994. Mathematical modeling of optical glazing performance. Proc. SPIE 2255, Optical Materials Technology for Efficiency and Solar Energy Conversion XIII, p. 753; doi: 10.1117/12.185417.

van Nijnatten, P.A, 1999. Predictive algorithms for directional optical properties of coated glazing for building and automotive applications. Thin Solid Films 351, pp. 295300 (1999); doi:10.1016/S0040-6090(99)00258-8

van Nijnatten, P.A., 2001. A pseudo-Fresnel approach for predicting directional optical properties of coated glazing. Thin Solid Films, 392, pp. 282-288; doi: 10.1016/S00406090(01)01044-6.

Wijeysundera, N.E, 1975. A net radiation method for the transmittance and absorptivity of a series of parallel regions. Solar Energy, 17, pp. 75-77.

Wright, J.L., 1998. Calculating centre-glass performance indices of windows. ASHRAE Transactions 104, Pt. 1, pp. 1230-1241. 
Yamada, T., Nakamura, H., Sugiura, T., Sakuta, K., Kurokawa, K., 2001. Reflection loss analysis by optical modeling of PV module. Solar Energy Materials \& Solar Cells 67, pp. 405-413. doi:10.1016/S0927-0248(00)00309-3 